\title{
Coherence of a Room-Temperature CW GaAs/GaAIAs Injection Laser
}

\author{
AXEL R. REISINGER, C. D. DAVID, JR., K. L. LAWLEY, AND AMNON YARIV, FELLOW, IEEE
}

\begin{abstract}
The temporal coherence of a stripe-geometry doubleheterojunction $\mathrm{GaAs} / \mathrm{GaAlAs}$ laser operating $\mathrm{CW}$ at room temperature was determined. A heterodyne detection scheme was used involving the mixing of the laser field with a frequency-shifted and time-delayed image of itself in an interferometer. Because the laser device oscillated in several longitudinal modes, the autocorrelation function of its output exhibited resonances for specific time delays. The rate at which the amplitude of these resonances decreased with increasing time delays provided a measure of an apparent coherence length associated with individual longitudinal modes. The coherence length, so defined, was found to increase linearly with drive current in excess of threshold. This observation is interpreted as evidence that the intrinsic linewidth of a longitudinal mode is inversely proportional to the coherent optical power in that mode. Apparent coherence lengths were a few centimeters for a few milliwatts of total optical power emitted per facet. For a perfectly balanced interferometer, a sharp heterodyne beat signal was also observed when the laser device was operated considerably below threshold, i.e., in the LED mode.
\end{abstract}

\section{INTRODUCTION}

T HE COHERENCE properties of conventional GaAs injection lasers have been investigated by a number of authors [1]-[8]. All of these experiments were concerned with homojunction devices operated either $\mathrm{CW}[1],[3],[4]$, [8] or pulsed [2], [5]-[7] at liquid nitrogen temperature [2], [4]- [8] or below [1], [3]. The intrinsic laser linewidth was determined by several methods, including the use of a Fabry-Perot interferometer [1], heterodyne mixing of adjacent longitudinal modes in large laser cavities [2], [3], and homodyne detection of correlated noise in an unbalanced Michelson interferometer [4]. Spectral information can also be obtained indirectly from measurements of the temporal coherence of the laser by holographic techniques [5]-[8], which amount to a determination of the visibility of interference fringes in an unbalanced interferometer. The best reported result on a GaAs homojunction laser was a linewidth substantially smaller than $150 \mathrm{kHz}$ (the detection system's resolution) for a device operated CW at $77 \mathrm{~K}$ with $250 \mathrm{~mW}$ of optical power emitted in a single mode [4].

This paper describes measurements of the coherence length of a GaAs/GaAlAs double-heterojunction stripe-geometry multimode laser operated $\mathrm{CW}$ at room temperature with an

Manuscript received April 4, 1979; revised July 5, 1979.

A. Reisinger was with Texas Instruments, Inc. Dallas TX. He is now with Optical Information Systems, Exxon Enterprises, Inc., Elmsford, NY 10523.

C. D. David, Jr. and K. L. Lawley are with Texas Instruments, Inc., Dallas, TX 75222.

A. Yariv is with the Department of Electrical Engineering, California Institute of Technology, Pasadena, CA 91109.

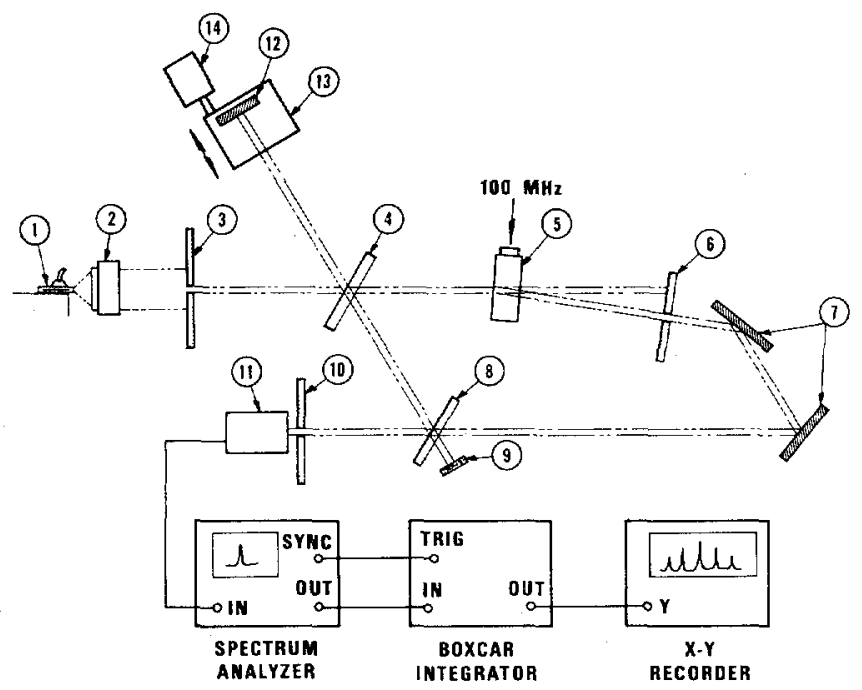

Fig. 1. Schematic of the experimental apparatus: $1=1$ laser, $2=10 \mathrm{X}$ microscope objective, 3,6 , and $10=$ apertures, 4 and $8=$ beam splitters, $5=$ acoustooptic Bragg cell, $7=$ stationary mirrors, $9=$ beam stop, $11=$ photomultiplier, $12=$ movable mirror, $13=$ translation stage, and $14=$ electric motor drive.

optical power output of a few milliwatts per facet. The experimental techniques used involved heterodyne mixing of the laser field with a frequency shifted image of itself in an unbalanced interferometer.

\section{Experimental Apparatus}

A schematic of the experimental apparatus is shown in Fig. 1. The output of the laser source is collimated by means of a $10 \mathrm{X}$ microscope objective, reduced in diameter by an aperture, and divided into two arms by a first beam splitter. One arm goes through an acoustooptic Bragg cell operated at a frequency of $100 \mathrm{MHz}$. An aperture is used to pass only the Braggdiffracted light beam, which has been frequency shifted by the acoustic frequency. This beam is then redirected by means of two stationary mirrors toward a detector. The second arm of the interferometer is reflected back by a movable mirror, traverses the first beam splitter, and is recombined with the first arm by means of a second beam splitter. The detector is a photomultiplier with an $S-1$ response (RCA-7102). It sees the superposition of the laser field with a frequency shifted image of itself. In this sense, the experiment amounts to a heterodyne detection scheme, with the laser field playing the role of local oscillator, and the frequency shifted field acting as the signal [9]. The purpose of the movable mirror mounted on a translation stage, which is driven by an electric motor, is 


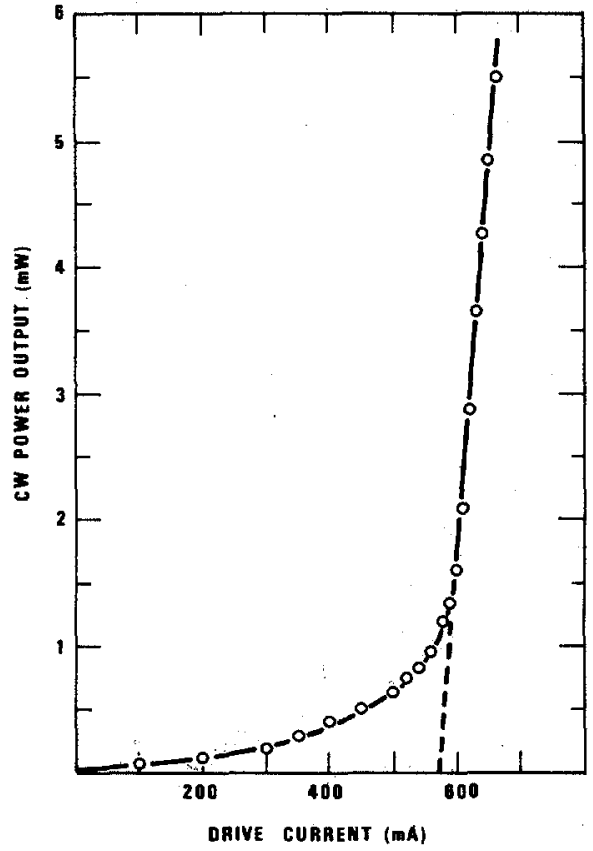

Fig. 2. Laser power output (one side) versus drive current for the laser device used in these experiments.

to introduce an adjustable path length difference between the two arms of the interferometer. The photocurrent generated in the photomultiplier is displayed on a spectrum analyzer (Tektronix, Type 491).

The injection laser used was a double-heterostructure stripegeometry room-temperature $\mathrm{CW} \mathrm{GaAs/GaAlAs} \mathrm{laser} \mathrm{fabricated}$ at Texas Instruments. The optical power output versus drive current of this particular device is shown in Fig. 2. In spite of the relatively high-threshold current of $575 \mathrm{~mA}$, no appreciable degradation was observed during the course of the experiment (estimated operation $\sim 250 \mathrm{~h}$ ). As evidenced by the spectrum (taken $25 \mathrm{~mA}$ above threshold) shown in Fig. 3, the laser oscillated in a large number of longitudinal modes. Laser emission peaked around $8900 \AA$, and the thode spacing was about $2.7 \AA$, consistent with a Fabry-Perot cavity length of $300 \mu \mathrm{m}$.

\section{Results}

It was found that the amplitude of the heterodyne beat signal at $100 \mathrm{MHz}$ depended very critically on the position of the movable mirror. The effect can be dramatically demonstrated by slowly scanning the mirror position with the electric motor, monitoring the amplitude of the beat signal with a boxcar integrator, and displaying it directly on a $X-Y$ recorder. The result is shown in Fig. 4. The existence of the resonances for discrete mirror positions separated by about $1.5 \mathrm{~mm}$ is directly traceable to the multimode nature of the laser [6], [10]. Each longitudinal mode of Fig. 3 generates its own beat signal at $100 \mathrm{MHz}$. However, there is a phase difference $\Delta \phi$ between the contributions to the beat signal from two adjacent longitudinal modes, since they oscillate at different frequencies. This phase difference can be written as

$$
\Delta \phi=\Delta k \cdot(2 x)
$$

where $2 x$ is the path length difference between the two arms

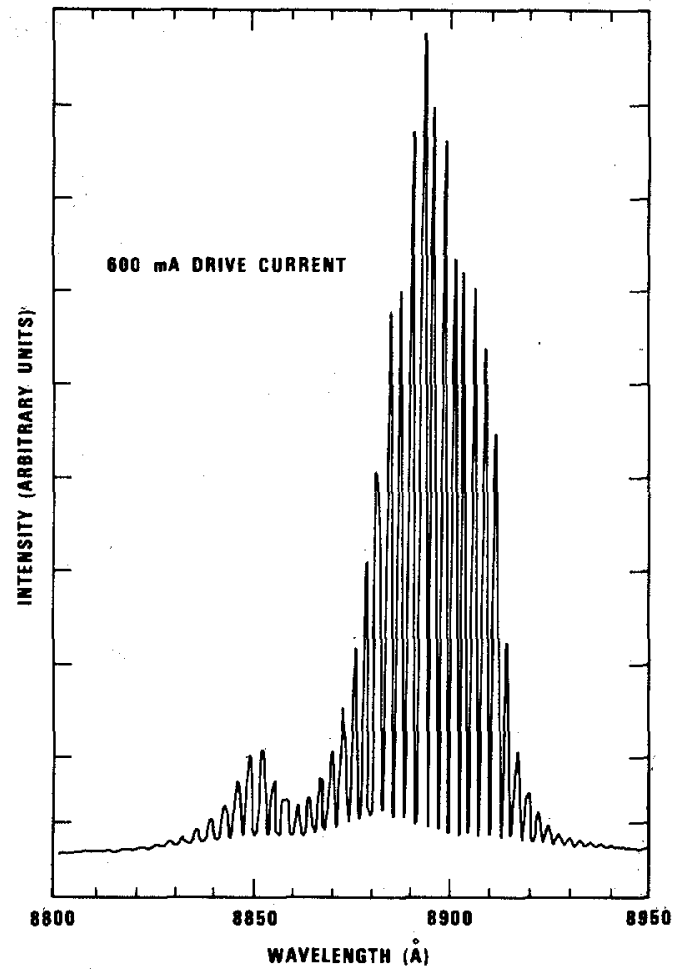

Fig. 3. Output spectrum of the laser $25 \mathrm{~mA}$ above threshold.

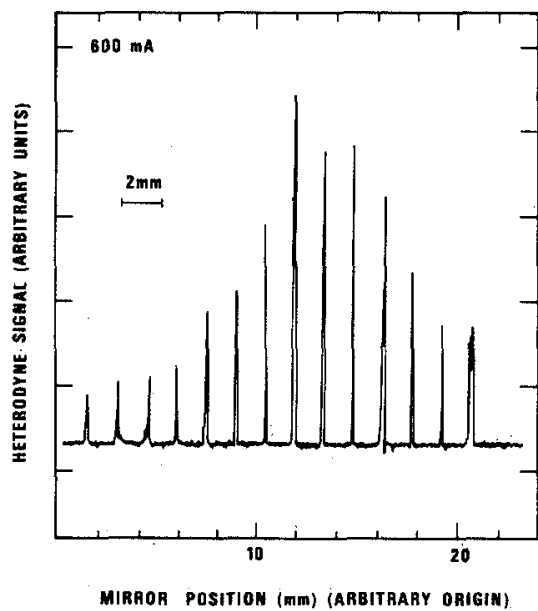

Fig. 4. Amplitude of heterodyne beat signal $(100 \mathrm{MHz})$ versus position of movable mirror.

of the interferometer and $\Delta k$ is the wave-vector difference between two adjacent longitudinal modes. The factor of 2 comes about because when the mirror is displaced by an amount $x$, the path length imbalance changes by twice that amount. The contributions to the beat signal arising from the individual laser modes will add up cooperatively only when they are all in phase, i.e., when $\Delta \phi$ of (1) obeys the relation

$$
\Delta \phi=2 N \pi
$$

where $N$ is an integer. The quantity $\Delta k$ and the longitudinal mode spacing $\Delta \lambda$ are related by

$$
\Delta k=2 \pi \Delta \lambda / \lambda^{2} .
$$


Equations (1)-(3) imply thát a strong beat signal will be observed for mirror positions s̀eparated by

$$
\Delta x=\lambda^{2} /(2 \Delta \lambda) \text {. }
$$

With $\lambda=8900 \AA$ and $\Delta \lambda=2.7 \AA$ in (4), the spatial periodicity $\Delta x$ is $1.47 \mathrm{~mm}$, in good agreement with the experimentally observed value. One expects the sharpness of the resonances as a function of mirror position to increase with the number of longitudinal modes.

It is shown in Apperidix $I$ that the magnitude of the component $i_{\Omega}$ of the photocurrent at angular frequency $\Omega$ is proportional to the amplitude of the autocorrelation function $g_{E}^{(1)}(\tau)$ of the laser field

$$
i_{\Omega} \propto\left|g_{E}^{(1)}(\tau)\right| \text {. }
$$

It is further shown that for a multimode laser, whose individual modes have a Lorentzian profile, the autocorrelation function can be written in the form

$$
g_{E}^{(1)}(\tau)=\sum_{k} A_{k} \exp -j \omega_{k} \tau \exp -\gamma_{k}|\tau|
$$

where each subscript $k$ refers to a particular longitudinal mode oscillating at angular frequency $\omega_{k}$, with a half-width at halfpower of $\gamma_{k}$. The coefficients $A_{k}$, which are a measure of the amount of coherent optical power in mode $k$, are normalized so that

$$
\sum_{k} A_{k}=1 \text {. }
$$

Equation (6) is obtained by taking the Fourier transform of the power spectral density of a multimode laser source (WienerKhintchine theorem [11]). If for simplicity we assume that all the modes have the same spectral width $\gamma_{o},(6)$ reduces to

$$
g_{E}^{(1)}(\tau)=\exp =\gamma_{o}|\tau| \sum_{k} A_{k} \exp -j \omega_{k} \tau
$$

The summation over $k$ is, of course, responsible for the resonant behavior exemplified by the result of Fig. 4. The amplitude of $g_{E}^{(1)}(\tau)$ is maximum when all the exponential terms in the summation are in phase. For regularly spaced modes, the condition is

$$
\Delta \omega \cdot \tau=2 \pi
$$

where $\Delta \omega$ is the angular frequency difference between adjacent modes. Since $\tau=2 x / c$ ( $c=$ speed of light), it is trivial to show that the above condition is equivalent to (4). When this condition is met, the beat signal $i_{\Omega}$ as expressed in (5), is maximum and given by

$$
\left(i_{\Omega}\right)_{\max } \propto \exp -\gamma_{o}|\tau| \text {. }
$$

Referring to Fig. 4, the envelope of the peaks does appear to go through a maximum (corresponding to a balanced interferometer) and to decay on either side of this maximum, in qualitative agreement with (10). While the continuous scan experiment of Fig. 4 demonstrates the sharpness of the spatial resonances, accurate measurements of the maximum ampli-

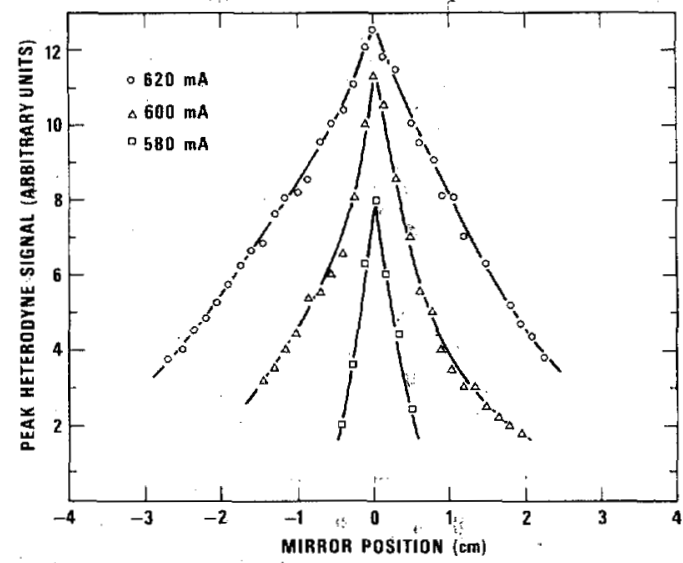

Fig. 5. Envelope of the peak signal versus position of the movable mirror for drive currents of 580,600 , and $620 \mathrm{~mA}$, respectively. Position 0 corresponds to balanced interferometer.

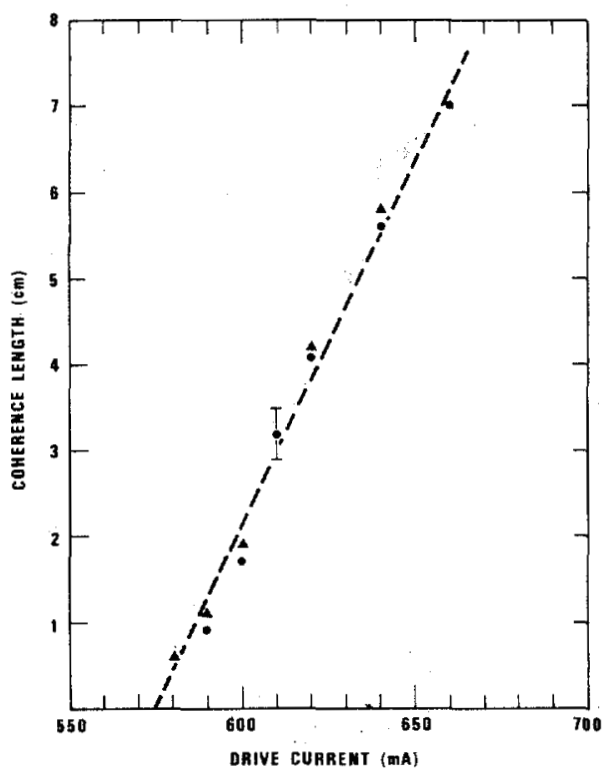

Fig. 6. Coherence length versus drive current. The circles and triangles correspond to two sets of measurements.

tude of each peak require that the position of the movable mirror be tuned manually and its orientation optimized for each peak. Such careful measurements were made at several drive currents. Peak amplitudes versus mirror position are shown in Fig. 5 for drive currents of 580,600 , and $620 \mathrm{~mA}$, respectively. The width of the envelope increases with increasing drive current. A coherence length can be defined as the distance at which the envelope drops by a factor of $1 / e$ from its maximum value (with balanced interferometer). Fig. 6 is a plot of the coherence length, defined in this manner, versus drive current. The plot appears linear and intersects the horizontal axis at or near the threshold current of $575 \mathrm{~mA}$. Since coherence length and frequency bandwidth are inverse of each other, and since laser output is--to a first approximationproportional to drive current in excess of threshold, Fig. 6 is interpreted as evidence that the intrinsic linewidth of the longitudinal modes is inversely proportional to the $\mathrm{CW}$ optical laser output $[1],[12]$. A reduction of the linewidth, with 
increasing drive current, has been demonstrated previously in PbSnTe CW lasers operating at $4.2 \mathrm{~K}$ [12].

\section{Discussion}

Possible sources of error that would cause erroneous measurements of the coherence length are 1) misalignment of the two interfering beams, 2) nonuniform linewidth for the various longitudinal modes, and 3) unequal frequency spacing between adjacent longitudinal modes over the emission spectrum.

With a He-Ne laser we could observe a beat signal whose amplitude was constant within 20 percent for path length differences up to $50 \mathrm{~cm}$. Our alignment procedure thus appears sufficiently accurate to eliminate this potential problem.

Rather than assigning the same spectral width $\gamma_{o}$ to all longitudinal modes, it is more reasonable to assume that the width of each longitudinal mode is inversely proportional to the amount of coherent optical power in that mode [13]. Thus, we write

$$
\gamma_{k}=\gamma_{o}\left(A_{o} / A_{k}\right)
$$

where the subscript $o$ refers to the dominant longitudinal mode. Each coefficient $A_{k}$ can be evaluated from the spectral data of Fig. 3 as being proportional to the area under the peak corresponding to mode $k$, subject to the normalization condition of (7). We can anticipate that this correction will tend to shorten the "apparent" coherence length, although not by a very large amount, since the broadest modes are also the weakest.

Perhaps more important is the effect of unequal spacing in the frequency domain between adjacent pairs of longitudinal modes. This second-order dispersion phenomenon has been used previously to determine the group index of water [14] The average frequency spacing between adjacent longitudinal modes in the present laser was $\delta \nu \simeq 100 \mathrm{GHz}$. The magnitude of the second-order dispersion $\delta^{(2)}(\nu)$ is usually 50 $100 \mathrm{MHz}[15]$. Since $\delta^{(2)}(\nu)$ can be defined by

$$
\delta^{(2)}(\nu)=\left(\nu_{k+1}-\nu_{k}\right)-\left(\nu_{k}-\nu_{k-1}\right)
$$

it is easy to show recursively that

$$
\omega_{k}-\omega_{o} \simeq k 2 \pi \delta \nu+k^{2} \pi \delta^{(2)}(\nu)
$$

where the subscripts $o$ and $k$ have the same meaning as in (11). The influence of both nonuniform linewidth and secondorder dispersion can be ascertained by substituting (11) and (13) into (6). The result is

$$
\begin{aligned}
\left|g_{E}^{(1)}(\tau)\right|= & \exp -\gamma_{o} \tau \mid \sum_{k} A_{k} \exp j\left(\omega_{k}-\omega_{o}\right) \tau \\
& \cdot \exp -\gamma_{o}\left[\left(A_{o} \mid A_{k}\right)-1\right] \tau \mid .
\end{aligned}
$$

Fig. 7 is a plot of the magnitude of the autocorrelation function versus mirror position, calculated from (14) with the following parameter values: $\gamma_{o}=1.5 \times 10^{10} \mathrm{rad} / \mathrm{s}\left(L_{\text {coh }}=\right.$ $\left.c / \gamma_{o}=2 \mathrm{~cm}\right)$ and $\delta^{(2)}(\nu)=50 \mathrm{MHz}$. The relative mode amplitudes $A_{k}$ were determined on the basis of Fig. 3. If the contribution of the dominant peak alone, represented by the dotted curve, is used to define the "intrinsic" coherence length $L_{\text {coh }}$, the "apparent" coherence length $L_{\text {coh }}^{\prime}$ is reduced
GAMMA $-1.5 E+10$ RAD/SEC

$D N U(2)=50 \mathrm{MHTZ}$

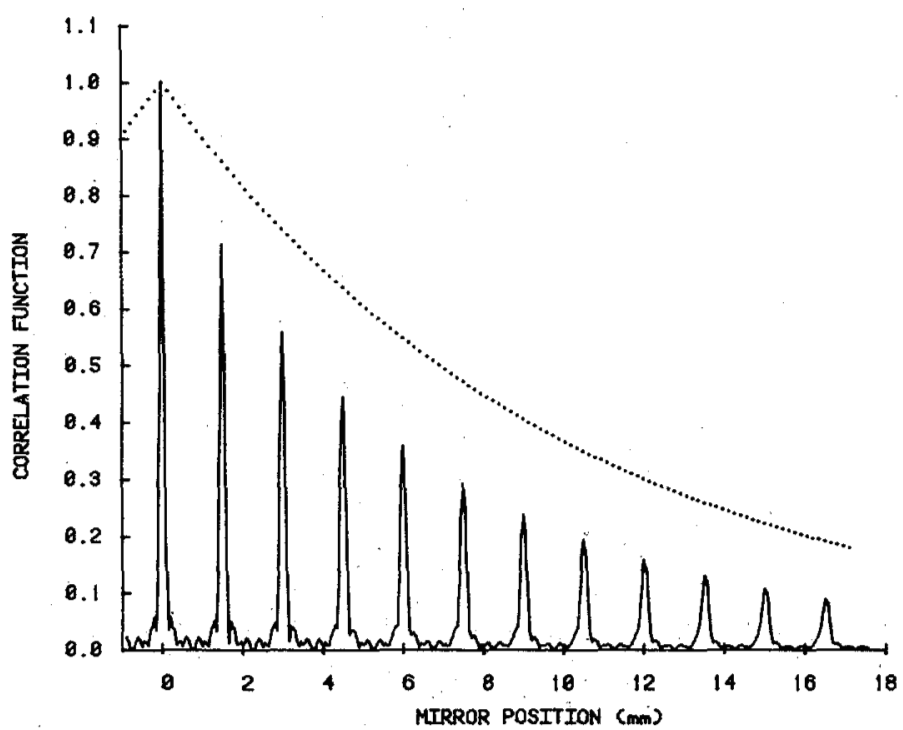

Fig. 7. Computer plot of the magnitude of the laser field autocorrelation function versus mirror position calculated from the spectrum of Fig. 3. The dotted curve is the contribution of the dominant peak alone.

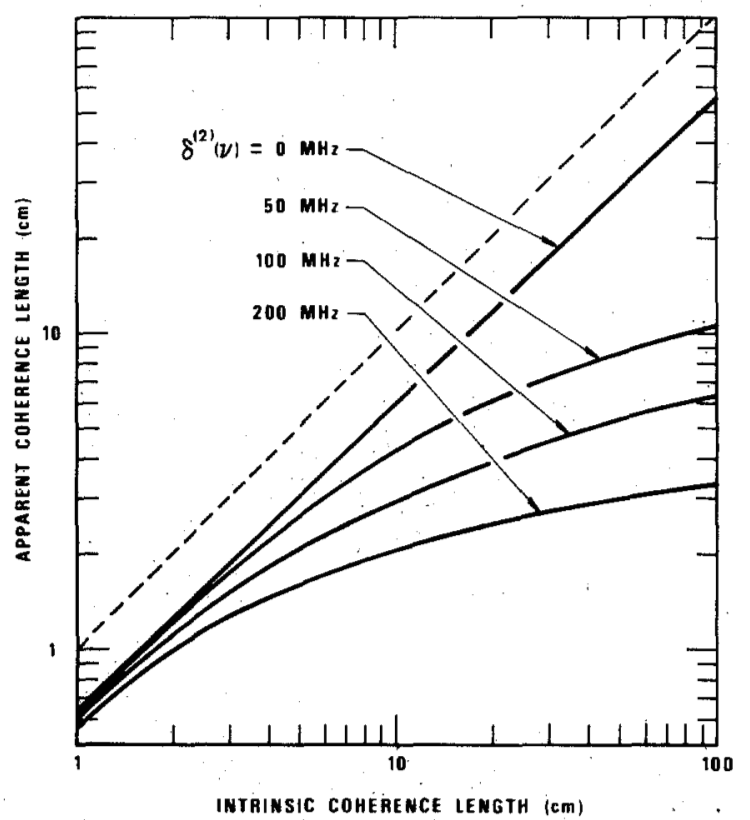

Fig. 8. "Apparent" coherent length versus "intrinsic" coherent length illustrating the effect of nonuniform linewidth and increasing secondorder dispersion $\delta{ }^{(2)}(\nu)$.

in this particular case by a factor of about 1.6. Fig. 8 is a log$\log$ graph of $L_{\text {coh }}^{\prime}$ plotted against $L_{\text {coh }}$ for various conditions. The straight dotted line with slope 1 corresponds to a uniform linewidth $\gamma_{o}$ for all modes and no second-order dispersion. In this case, $L_{\text {coh }}$ and $L_{\text {coh }}^{\prime}$ are equal in the sentse that the envelope of the correlation peaks as a function of time delay $\tau$ coincides with the correlation function associated with the dominant longitudinal mode alone. The straight solid line includes the effect on nonuniform linewidth only (but still no second-order dispersion). The "apparent" coherence length is 
reduced by a factor of about 1.6. The solid curves in Fig. 8 show the additional contribution of second-order dispersion, which causes a further shortening of the apparent coherence length. The effect is most noticeable for long intrinsic coherence lengths and large values of the parameter $\delta^{(2)}(\nu)$. Of interest is the fact that for large intrinsic linewidths $\Delta \nu_{o}$ (or short $L_{\mathrm{coh}}$, since $\left.L_{\mathrm{coh}}=c / \pi \Delta \nu_{o}\right)$, second-order dispersion is relatively unimportant. The implication of Fig. 8 is that if indeed $L_{\text {coh }}^{\prime}$ increases in proportion to coherent optical power output, as the theory predicts, $L_{\text {coh }}$ at first should increase linearly with drive current and eventually should tend to saturate. No evidence of saturation was observed in the present experiment. It should be emphasized, however, that Fig. 8 was obtained specifically with the spectrum of Fig. 3 and assumes, rather restrictively, that this spectrum remains independent of lasing conditions. A more thorough quantitative analysis would require a detailed knowledge of the evolution of the coherent emission spectrum as a function of drive current. With these restrictions in mind, one infers from Figs. 7 and 8 an "intrinsic" coherence length of order of magnitude $10 \mathrm{~cm}$ at a power output level of $\simeq 5 \mathrm{~mW} /$ facet. This corresponds to an "intrinsic" linewidth of $\simeq 1 \mathrm{GHz}$. On the basis of Fig. 3 it is estimated that about 7 percent of the total optical power is contained in one of the dominant longitudinal modes. One concludes that if the total power were emitted in a single longitudinal mode, the linewidth at that power level would be expected to be around $70 \mathrm{MHz}$.

It is interesting to note in passing that, when the mirror position is adjusted for a perfectly balanced interferometer, the beat signal continues to be observed with a drive current as low as $350 \mathrm{~mA}$. This current is considerably below threshold (see Fig. 2), and the radiation emitted by the device is then essentially incoherent and very broad-band, covering about $200 \AA$. Accordingly, the coherence length is extremely small, and the beat signal disappears as soon as the interferometer is detuned. Furthermore, with incoherent light, the width of the beat signal as seen on the spectrum analyzer continues to be as narrow as that observed when the device is operated in the laser mode (the bandwidth appears limited by the electronics driving the acoustooptic modulator). This initially surprising result can in fact be understood if one recognizes that the response of a photomultiplier (a square law detector) to a broad-band incoherent-optical field (one which obeys Gaussian statistics) [11] gives rise to a photocurrent whose spectrum consists of a delta function at zero frequency (a strong dc component) superposed on a broad background [16], [17]. In the present heterodyne detection scheme, the optical field is mixed not only with itself but also with an image of itself, which has been frequency shifted by $100 \mathrm{MHz}$. One would then expect the photocurrent spectrum to display an additional delta function at $100 \mathrm{MHz}$. A proof of this intuitive argument is outlined in Appendix II. It is this additional delta function (in practice a narrow peak) which was observed. Heterodyne mixing of incoherent light beams has been demonstrated previously [18].

\section{SUMmary}

The apparent coherence length of a stripe-geometry doubleheterojunction GaAs/GaAlAs laser operated $\mathrm{CW}$ at room temperature was measured by a heterodyne detection scheme which involves mixing the laser field with a frequency-shifted image of itself. Because of the multimode nature of the laser output, the autocorrelation function of the optical field, as determined in an unbalanced interferometer, displayed a resonant behavior for specific time delays. The apparent coherence length was shown to increase linearly with drive current in excess of threshold. This observation is interpreted as evidence that the intrinsic linewidth is inversely proportional to the coherent optical power output.

\section{APPENDIX I \\ Photocurrent and Autocorrelation function OF OPTICAL FiELD}

The total optical field $E_{T}$ incident on the photocathode of the detector can be written as

$$
E_{T}(t)=E(t)+\exp j \Omega t E(t+\tau)
$$

where the first term represents the time dependence of the laser field, and the second term describes its image shifted in frequency by the acoustic angular frequency $\Omega$ and delayed in time by the amount $\tau$ corresponding to the imbalance between the two arms of the interferometer. For simplicity, both fields have been assumed to have equal amplitudes. The instantaneous photocurrent $i(t)$ is proportional to the total field intensity $E_{T}(t) E_{T}^{*}(t)$ where the star * denotes the complex conjugate

$$
i(t) \propto E_{T}(t) E_{T}^{*}(t) .
$$

Substitution of (15) into (16) gives rise to four terms, as is common in all interference problems. In paiticular, one of the two cross terms represents the instantaneous photocurrent component $i_{\Omega}(t)$ at angular frequency $+\Omega$

$$
i_{\Omega}(t) \propto E^{*}(t) E(t+\tau) \exp j \Omega t .
$$

An interference experiment of the type discussed here can only provide a measure of the statistical average $\left\langle i_{\Omega}\right\rangle$ of the amplitude of the photocurrent at frequency $\Omega$ :

$$
\left\langle i_{\Omega}\right\rangle \propto\left\langle E^{*}(t) E(t+\tau)\right\rangle .
$$

The right-hand side of (18) is related to the first-order (complex) autocorrelation function $g_{E}^{(1)}(\tau)$ of the laser field alone, which is defined as

$$
g_{E}^{(1)}(\tau)=\left\langle E(t) E^{*}(t+\tau)\right\rangle /\left\langle E(t) E^{*}(t)\right\rangle
$$

It follows that the average magnitude of the photocurrent component at angular frequency $\Omega$ is given by

$$
\left|\left\langle i_{\Omega}\right\rangle\right| \propto\left|g_{E}^{(1)}(\tau)\right| \text {. }
$$

The power spectral density of a single-laser mode with a Lorentzian profile is

$$
\phi(\omega)=\frac{\gamma_{0} / \pi}{\gamma_{0}^{2}+\left(\omega-\omega_{0}\right)^{2}}
$$

Application of the Wiener-Khintchine theorem gives

$$
g_{E}^{(1)}(\tau)=\exp -j \omega_{0} \tau \exp -\gamma_{0}|\tau|
$$

For $K$ distinct laser modes, each with Lorentzian profile, the power spectral density becomes 


$$
\phi(\omega)=\sum_{k=1}^{K} A_{k} \frac{\gamma_{k} / \pi}{\gamma_{k}^{2}+\left(\omega-\omega_{k}\right)^{2}}
$$

In order to normalize the power spectral density $\left(\int_{-\infty}^{+\infty} \phi(\omega)\right.$ $d \omega=1$ ) the coefficients $A_{k}$ are subject to the restriction

$$
\sum_{K} A_{k}=1
$$

By taking the inverse Fourier transform of (23) the autocorrelation function of the laser field now reads

$$
g_{E}^{(1)}(\tau)=\sum_{k} A_{k} \exp -j \omega_{k} \tau \exp -\gamma_{k}|\tau|
$$

\section{APPENDIX II \\ Photocurrent Spectrum}

By virtue of the Wiener-Khintchine theorem, the spectrum of the photocurrent is given by the Fourier transform of its autocorrelation function $C_{i}(\tau)$, which, by definition, is

$$
C_{i}(\tau) \propto\langle i(t) i(t+\tau)\rangle=\left\langle E_{T}(t) E_{T}^{*}(t) E_{T}(t+\tau) E_{T}^{*}(t+\tau)\right\rangle .
$$

It is apparent from (26) that the photocurrent autocorrelation function is proportional to the second-order correlation function of the total optical field [17]

$$
C_{i}(\tau) \propto g_{E}^{(2)}(\tau) .
$$

Substitution of (15) into (26) gives rise to 16 terms. Ten of those 16 terms, namely those in which a time dependence appears explicitly in the form $e^{ \pm j \Omega t}$, average out to 0 . This follows from the fact that, since we are dealing with stationary fields, the ensemble and time averages are identical. The surviving six terms give the result

$$
C_{i}(\tau)=g_{E}^{(2)}(\tau) \times(4+\exp i \Omega \tau+\exp -j \Omega \tau) / 6 .
$$

For fully coherent light, $g_{E}^{(2)}(\tau)=1$. The power spectrum $P_{i}(\omega)$ of the photocurrent is obtained by taking the Fourier transform of $(28)$ with $g_{E}^{(2)}(\tau)=1$

$$
P_{i}(\omega)=(1 / 6)[4 \delta(\omega)+\delta(\omega+\Omega)+\delta(\omega-\Omega)] .
$$

The photocurrent spectrum is made up of $\delta$-functions centered at $\omega=0$ (the $\mathrm{dc}$ term) and $\omega= \pm \Omega$ (positive and negative frequencies are equivalent in this analysis).

For incoherent light described by random Gaussian fields [11], the second-order correlation function can be expressed in terms of the first-order correlation function [17]

$$
g_{E}^{(2)}(\tau)=1+\left|g_{E}^{(1)}(\tau)\right|^{2} .
$$

The first term (equal to 1 ) will clearly produce the same photocurrent power spectrum [see (29)] as in the case of the fully coherent field. The second term $\left(\left|g_{E}^{(1)}(\tau)\right|^{2}\right)$ gives rise to additional broad background spectra centered at $\omega=0$ and $\omega= \pm \Omega$. These additional contributions constitute the excess noise which disappears for coherent light [1] .

\section{ACKNOWLEDGMENT}

We are grateful to D. W. Bellavance for supplying the laser material used in this work, to W. Abercrombie for device fabrication and to $S$. Slingerland for assistance in some phases of the experiment. A. Reisinger wishes to thank F. Sutherland as well as E. Chua of OIS for help with the computer calculations and acknowledges useful conversations with D. J. Coleman, W. C. Scott, and M. deWit. Credit must be given to an anonymous reviewer for pointing out the potential importance of second order dispersion.

\section{REFERENCES}

[1] J. A. Armstrong and A. W. Smith, "Interferometric measurement of linewidth and noise in GaAs lasers," Appl. Phys. Lett., vol. 4, pp. 196-198, June 1, 1964.

[2] J. W. Crowe and R. M. Craig, Jr., "GaAs laser linewidth measurements by heterodyne detection," Appl. Phys. Lett., vol. 5, pp. 72-74, Aug. 15, 1964.

[3] M. Ciftan and P. P. Debye, "On the parameters which affect the C. W. output of GaAs lasers," Appl. Phys. Lett., vol. 6, pp. 120121, Mar. 15, 1965.

[4] W. E. Ahearn and J. W. Crowe, "Linewidth measurements of CW gallium arsenide lasers at $77^{\circ} \mathrm{K}, "$ IEEE J. Quantum Electron., vol. QE-2, pp. 597-602, Sept. 1966.

[5] Yu. A. Bykovskii, V. A. Elkhov, and A. I. Larkin, "Coherence of the radiation emitted by a semiconductor laser and its use in holography," Sov. Phys.-Semicond., vol. 4, pp. 819-821, Nov. 1970.

[6] Yu. A. Bykovskii et al., "Coherence of the radiation of a pulsed single-mode injection semiconductor laser," Sov. Phys.-Dokl., vol. 17, pp. 359-361, Oct. 1972.

[7] Yu. A. Bykovskii et al., "Single-pulse holography employing injection lasers," Sov. J. Quantum Electron., vol. 4, p. 136, July 1974.

[8] Yu. M. Mironov, V. I. Molochev, V. V. Nikitin, and A.S.Semenov, "Investigation of the emission line width of an injection laser in strong and weak fields," Sov. J. Quantum Electron., vol. 6, pp. 123-124, Jan. 1976.

[9] H. Z. Cummins and N. Knable, "Single sideband modulation of coherent light by bragg reflection from acoustical waves," Proc. IEEE (Correspondence), vol. 51, p. 1246, Sept. 1963.

[10] L. Mandel and E. Wolf, "The measures of bandwidth and coherence time in optics," Proc. Phys. Soc., vol. 80, pp. 894-897, 1962.

[11] -, "Coherence properties of optical fields," Rev. Mod. Phys., vol. 37, pp. 231-287, A pr. 1965.

[12] E. D. Hinkley and C. Freed, "Direct observation of the Lorentzian line shape as limited by quantum phase noise in a laser above threshold," Phys. Rev. Lett., vol. 23, pp. 227-280, Aug. 11, 1969.

[13] P. P. Sorokin, J. D. Axe, Jr., and J. R. Lankard, "Spectral characteristics of continuously operating GaAs lasers," in Proc. Symp. Opt. Masers, vol. XIII. New York: Polytechnic, 1963, pp. 481489.

[14] H. G. Danielmayer and H. P. Weber, "Direct measurement of the group velocity of light," Phys. Rev. A, vol. 3, pp. 1708-1713, May 1971.

[15] C. A. Brackett, "Second-order dispersion in oscillating GaAs junction lasers," IEEE J. Quantum Electron., vol. QE-8, pp. 66-69, Feb. 1972.

[16] W. B. Davenport and W. L. Root, Random Signals and Noise. New York: McGraw-Hill, 1963, ch. 12.

[17] H. Z. Cummins and H. L. Swinney, "Light beating spectroscopy," in Progress in Optics, E. Wolf, Ed., vol. XIII. Amsterdam: North-Holland, 1967.

[18] A. T. Forrester, R. A. Gudmundsen, and P. O. Johnson, "Photoelectric mixing of incoherent light;" Phys. Rev., vol. 99, pp. 1691-1700, Sept. 15, 1955. 